\title{
Experimental and Theoretical Studies of Free and Acceptor-Bound Positive Magneto-Trions
}

\author{
L. Bryja ${ }^{a}$, A. Wójs ${ }^{a}$, P. PŁochockA-Polack ${ }^{b}$,
} A. GŁadysiewicz ${ }^{a}$, J. Misiewicz ${ }^{a}$ And M. Potemski ${ }^{b}$

${ }^{a}$ Institute of Physics, Wrocław University of Technology

Wybrzeże Wyspiańskiego 27, 50-370 Wrocław, Poland

${ }^{b}$ Grenoble High Magnetic Field Laboratory, CNRS

38042 Grenoble Cedex 9, France

By combination of polarization-resolved photoluminescence, transport, and realistic numerics we study energy and recombination spectra of free and acceptor-bound positive trions in a quasi-two-dimensional hole gas. The singlet-triplet crossing in the trion ground state is found at $B \approx 12 \mathrm{~T}$, and a slight reduction of all trion binding energies coincident with the formation of a Laughlin hole fluid is observed at $B \approx 14.2 \mathrm{~T}$.

PACS numbers: 71.55.Cn, 71.35.Ji, 73.21.Fg

\section{Introduction}

Negative and positive trions $\left(\mathrm{X}^{ \pm}\right)$are bound states of an exciton $(\mathrm{X}=\mathrm{e}+\mathrm{h})$ and an additional carrier (e or h). These complexes often determine photoluminescence (PL) spectra of charged low-dimensional semiconductor nanostructures $[1,2]$. The energy spectrum of a trion confined in a quasi-two-dimensional $(2 \mathrm{D})$ quantum well of sufficiently small width $w$ and subject to high magnetic field $B$ contains several bound states characterized by total spin of the pair of identical carriers (singlet or triplet) and relative angular momentum $M[3,4]$. In particular, the singlet state with $M=0\left(\mathrm{X}_{\mathrm{s}}^{ \pm}\right)$and the pair of triplet states with $M=-1$ $\left(\mathrm{X}_{\mathrm{td}}^{ \pm}\right)$and $M=0\left(\mathrm{X}_{\mathrm{tb}}^{ \pm}\right)$are identified in various calculations. For free trions, only the $M=0$ states are optically active ("bright"), while recombination of the $M \neq 0$ ("dark") states requires the breaking of the $2 \mathrm{D}$ translational symmetry (e.g., impurity scattering or localization).

In this paper we combine magneto-PL experiments and realistic configuration interaction calculations to study the energy and recombination spectra of 
positive magneto-trions in $p$-doped quantum wells. In contrast to the previous experiments revealing only the singlet state, we report the first experimental detection of the entire family of $\mathrm{X}_{\mathrm{s}}^{+}, \mathrm{X}_{\mathrm{td}}^{+}$, and $\mathrm{X}_{\mathrm{tb}}^{+}$positive trions (with the singlettriplet crossing found at $B \approx 12 \mathrm{~T}$ ). We also observed a slight reduction of the trion binding energy at $B=14.2 \mathrm{~T}$ coincident with the condensation of the holes into the incompressible $\nu=1 / 3$ Laughlin liquid. Besides a free-trion emission, we also observed the recombination spectrum of acceptor-bound trions (including weak shake-up transitions [5], allowed by the breaking of 2D translational symmetry, not discussed here in detail).

\section{Experiment}

Studied samples $\mathrm{S}_{1}$ and $\mathrm{S}_{2}$ were $\mathrm{GaAs} / \mathrm{Al}_{0.35} \mathrm{Ga}_{0.65} \mathrm{As}$ quantum wells of width $w=15 \mathrm{~nm}$, grown by molecular beam epitaxy and C-doped in the barrier (symmetrically on both sides). The hole concentrations $p_{1}=1.15$ and $p_{2}=1.89 \times 10^{11} \mathrm{~cm}^{-2}$ were determined from transport (quantum Hall effect) measurements. The mobility of the $2 \mathrm{D}$ holes measured at low temperature $T=4.2 \mathrm{~K}$ was $\mu \approx 1 \times 10^{5} \mathrm{~cm}^{2} /(\mathrm{V} \mathrm{s})$. The PL was excited by a $720 \mathrm{~nm}$ red line of titanium sapphire tunable laser below the energy gap in the barrier. The additional $514 \mathrm{~nm}$ green ion argon line exceeding the barrier gap was used to decrease the hole concentration. The spectra were investigated in liquid helium pumped down to $T=1.8 \mathrm{~K}$ and in high magnetic fields up to $B=23 \mathrm{~T}$ (i.e., at low Landau level (LL) filling factors down to $\nu=1 / 5$ ) with a small step $\Delta B=0.1 \mathrm{~T}$. We used the Faraday configuration with the linear polarizer and quarter-wave plate placed together with the sample in liquid helium. To switch between the $\sigma^{-}$and $\sigma^{+}$circular polarizations the magnetic field direction was reversed.

The PL spectrum of sample $\mathrm{S}_{1}$ (result for $\mathrm{S}_{2}$ is similar) recorded at $B=0$ is shown in Fig. 1a. A single line with an exponential low-energy tail is due to the recombination of a spin-singlet trion $\mathrm{X}_{\mathrm{s}}^{+}$(the only bound trion at $B=0$ ). We used a logarithmic scale to illustrate the change of slope at about $\delta=1 \mathrm{meV}$ away from the peak maximum. It is shown even more clearly in the inset (b), where we plot the derivative of a logarithmic PL intensity $I$, i.e., $I^{-1} \mathrm{~d} I(E) / \mathrm{d} E$. This effect is explained as follows as a consequence of the finite radius $k_{\mathrm{F}}$ of the $2 \mathrm{D}$ hole Fermi surface. In the recombination of a free $\mathrm{X}^{+}$its total initial momentum $\hbar k$ shared by the three bound particles must be equal to the momentum of the single left-over hole. Depending on whether $k$ is larger or smaller than $k_{\mathrm{F}}$, the hole is left either outside or inside the Fermi sphere, leading to an abrupt change in the process intensity. Hence, only when $k>k_{\mathrm{F}}$ (i.e., at the energies sufficiently far below the emission maximum in Fig. 1a) we can expect exponential decay of $I(E)$ [6]. From the position $\delta$ of the slope change in Figs. 1a and b one can estimate the hole Fermi energy $E_{\mathrm{F}}=\hbar k_{\mathrm{F}}^{2} / 2 m_{\mathrm{h}}=\delta /\left[1-\left(2+m_{\mathrm{e}} / m_{\mathrm{h}}\right)^{-1}\right] \approx 1.7 \mathrm{meV}$ (in good agreement with the estimate from the hole concentration $p_{1}$ ). A similar slope change in an $n$-doped CdTe quantum well has been discussed earlier in Ref. [7] (in which also the thermal variation of the slope itself was explained). 


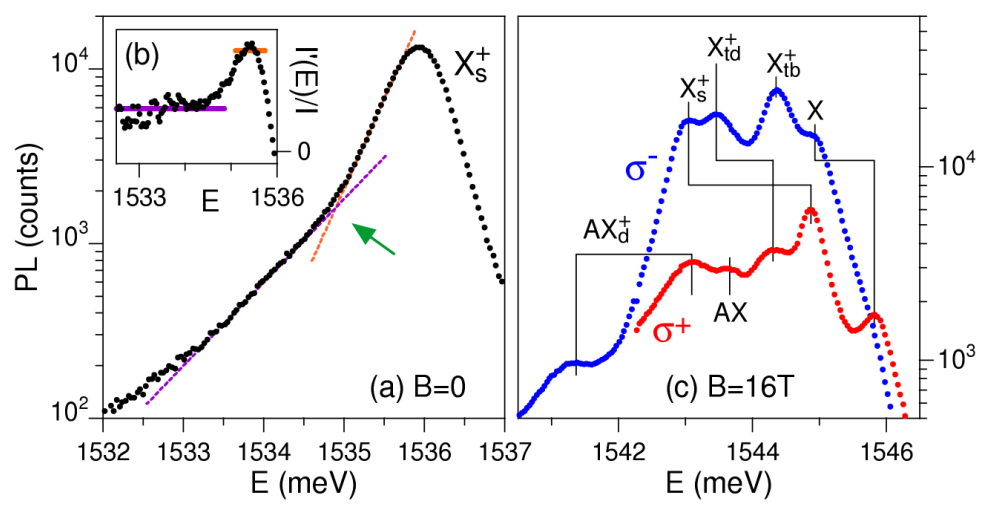

Fig. 1. PL spectra of a 2D hole gas at zero magnetic field (a) and at a high magnetic field $B=16 \mathrm{~T}$ (c). Inset (b): logarithmic field-derivative of the PL spectrum (a).

The polarization-resolved spectrum at $B=16 \mathrm{~T}$ is shown in Fig. 1c. Additional transitions, emergent only in high fields, are identified (in comparison with the numerics described further) as neutral exciton $(\mathrm{X})$, triplet trions $\left(\mathrm{X}_{\mathrm{td}}^{+}\right.$and $\mathrm{X}_{\mathrm{tb}}^{+}$), and neutral and charged acceptor-bound complexes ( $\mathrm{AX}$ and $\mathrm{AX}^{+}$). The whole family of three free positive trion states has been observed here for the first time.

In order to extract the Coulomb trion binding energy $\Delta=E[\mathrm{X}]+E[\mathrm{~h}]-$ $E\left[\mathrm{X}^{+}\right]$from the PL spectrum, the separations between the $\mathrm{X}$ and $\mathrm{X}^{+}$peaks must be averaged over both $\sigma^{+}$and $\sigma^{-}$polarizations. This is because of the different Zeeman splittings for the exciton and various trions, evident in Fig. 1c. The field evolution of $\Delta$ of $\mathrm{X}_{\mathrm{s}}^{+}$and $\mathrm{X}_{\mathrm{td}}^{+}$is displayed in Fig. 2a. The singlet-triplet crossing is found at $B \approx 12 \mathrm{~T}$ (relatively low field compared to the crossing of negative trions in typical $n$-doped wells).

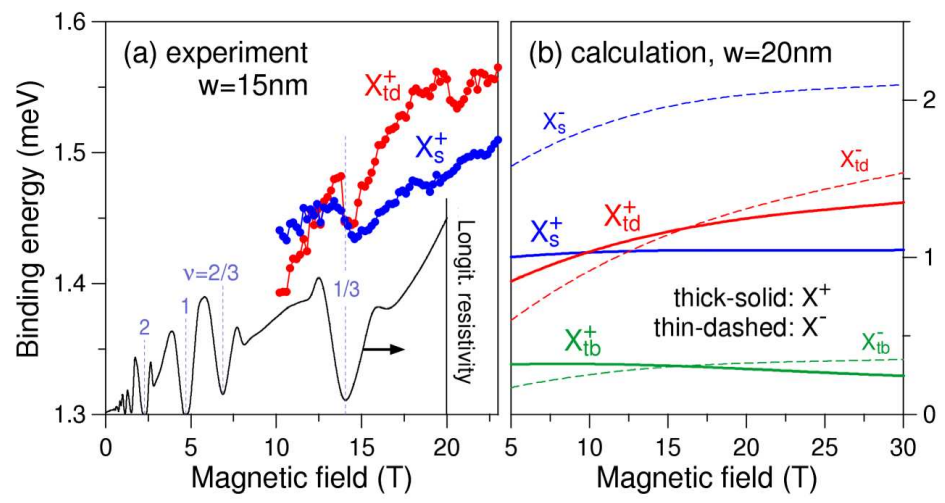

Fig. 2. Magnetic field dependence of experimental (a) and theoretical (b) positive trion binding energies $\Delta$. The quantum Hall curve also drawn in (a) allows one to identify the formation of the Laughlin hole liquid at $B \approx 14.2 \mathrm{~T}$. 
In Fig. 2a we also note downward cusps at $B=14.2 \mathrm{~T}$ in $\Delta$ of both singlet and triplet trions. From the quantum Hall measurements (see the longitudinal resistance curve $\left.\varrho_{x x}(B)\right)$ it is clear that $B=14.2 \mathrm{~T}$ corresponds to $\nu=1 / 3$, i.e., to the formation of a Laughlin incompressible hole fluid.

\section{Theory}

The identification of emission lines in Fig. 1 was possible by supplementing experiment with realistic numerical calculations of the Coulomb binding energies of various relevant excitonic complexes $\left(\mathrm{X}, \mathrm{X}^{+}, \mathrm{AX}\right.$, and $\left.\mathrm{AX}^{+}\right)$. The calculations consisted of exact diagonalization of the few-body e-h Hamiltonians in the Haldane spherical geometry, including five Landau levels and three quantum well subbands for both electrons and holes. In Fig. 2b we compare field dependence of $\Delta$ of different free negative and positive trions, calculated in a symmetric $w=20 \mathrm{~nm}$ quantum well. It is clear that the binding of the singlet trion weakens the most of all trions when going from $\mathrm{X}^{-}$to $\mathrm{X}^{+}$, leading to a significant shift of the singlettriplet crossing to a much lower magnetic field. Also, the dark triplet shows a much stronger field dependence than the pair of bright states. Similar calculations were also performed with an additional point charge (ionized acceptor) located inside the quantum well, in order to understand the $\mathrm{AX}$ and $\mathrm{AX}^{+}$recombination spectra, including a weak shake-up emission.

\section{Acknowledgments}

This work was partially supported by the Ministry of Science and Higher Education (Poland) grant N202-104-31/0771.

\section{References}

[1] K. Kheng, R.T. Cox, Y. Merle d'Aubigné, F. Bassani, K. Saminadayar, S. Tatarenko, Phys. Rev. Lett. 71, 1752 (1993).

[2] M. Byszewski, B. Chwalisz, D.K. Maude, M.L. Sadowski, M. Potemski, T. Saku, Y. Hirayama, S. Studenikin, D.G. Austing, A.S. Sachrajda, P. Hawrylak, Nature Physics (London) 2, 239 (2006).

[3] A. Wójs, J.J. Quinn, P. Hawrylak, Phys. Rev. B 62, 4630 (2000).

[4] G. Yusa, H. Shtrikman, I. Bar-Joseph, Phys. Rev. Lett. 87, 216402 (2001).

[5] S. Glasberg, H. Shtrikman, I. Bar-Joseph, Phys. Rev. B 63, 201308 (2001).

[6] A. Esser, E. Runge, R. Zimmermann, W. Langbein, Phys. Rev. B 62, 8232 (2000).

[7] P. Kossacki, J. Phys., Condens. Matter 15, R471 (2003). 